Lyall, Francis and Larsen, Paul B, Space Law: A Treatise [Ashgate Publishing, Farnham, 2009, xii + 596pp, ISBN 978-0-7546-4390-6, £80 (h/bk)]

Mallat, Chibli, Introduction to Middle Eastern Law [Oxford University Press, Oxford, 2009, xxxix + 455pp, ISBN 978-0-19-956387-6, £24.99 (p/bk)]

Oda, Hiroshi, Japanese Law (Third Edition) [Oxford University Press, Oxford, 2009, x +474pp, ISBN 978-0-19-923218-5, £85 (h/bk)]

Pan, Junwu, Towards a New Framework for Peaceful Settlement of China's Territorial and Boundary Disputes [Martinus Nijhoff Publishers, Leiden, Boston, 2009, viii +240pp, ISBN 978-90-04-17428-3, €99 (h/bk)]

Raue, Julia and Sutter, Patrick (eds), Facets and Practices of State-Building [Martinus Nijhoff Publishers, Leiden, Boston, 2009, vi + 340pp, ISBN 978-0-04-17403-0, € 110 (h/bk)]

Shany, Yuval, Regulating Jurisdictional Relations Between National and International Courts [Oxford University Press, Oxford, 2009, xxvii + 216pp, ISBN 978-0-19-956385-2, £29.99 $(\mathrm{p} / \mathrm{bk})]$

ShubBer, Sami, The Law of Investment in Iraq [Martinus Nijhoff Publishers, Leiden, Boston, 2009, xiii + 248pp, ISBN 978-90-04-17286-9, € 115 \$184 (h/bk)]

Ssenyonjo, Manisuli, Economic, Social and Cultural Rights in International Law [Hart Publishing, Oxford and Portland, 2009, xiii + 536pp, ISBN 978-1-84113-915-9, price not given $(\mathrm{p} / \mathrm{bk})]$

Twining, WiLliam, General Jurisprudence: Understanding Law from a Global Perspective [Cambridge University Press, Cambridge, 2009, xx + 517pp, ISBN 978-0-521-73809-5, £35 $(\mathrm{p} / \mathrm{bk})]$

White, Nigel D, Democracy Goes to War: British Military Deployments under International Law [Oxford University Press, Oxford, 2009, xv + 314pp, ISBN 978-0-19-921859-2, £60 (h/bk)]

\title{
INTRA-EU INVESTMENT AGREEMENTS AND ARBITRATION: IS EUROPEAN COMMUNITY LAW AN OBSTACLE?-CORRIGENDUM
}

\author{
HanNo WeHLAND
}

doi:10.1017/S0020589309001067, Published by Cambridge University Press, April 2009.

The internal cross-references were incorrectly reproduced. The correct cross references in the article are as follows:

Fn 21: $\mathrm{p} 302$.

Fn 28: $\mathrm{p} 300$.

Fn 41: p 311 .

Fn. 44: pp 309 ff.

Fn. 47: p 318.

Fn 67: pp 299 ff.

Fn 98: $\mathrm{p} 311$.

Fn. 110: $\mathrm{n}(120)$.

Fn 123: p 304.

\section{Reference}

$\mathrm{H}$ Wehland, 'Intra-EU Investment Agreements and Arbitration: Is European Community Law An Obstacle?' (2009) 58 ICLQ 2 297-320. 